\title{
The stability of magnetized protostellar disks with the Hall effect and buoyancy
}

\author{
V. Urpin ${ }^{1,2}$ and G. Rüdiger ${ }^{1}$ \\ 1 Astrophysikalisches Institut Potsdam, An der Sternwarte 16, 14482 Potsdam, Germany \\ e-mail: gruediger@aip.de \\ 2 A. F. Ioffe Institute of Physics and Technology, 194021 St. Petersburg, Russia
}

Received 25 August 2004 / Accepted 5 March 2005

\begin{abstract}
The stability properties of cool protostellar disks are examined by use of the dispersion relation taking into account the Hall effect and buoyancy. Depending on the conditions, different types of instabilities can arise in different regions of the disk. In very low-ionized regions the instability associated with baroclinic effects of buoyancy is likely most efficient. The shearHall instability will be responsible for destabilization of regions with a weak magnetic field (parallel to the rotation axis) and with low conductivity ( $\Lambda<a_{\mathrm{e}}, \Lambda=\sigma B^{2} / \rho \Omega$ Elsässer number, $a_{\mathrm{e}}$ magnetization parameter of electrons). The magnetorotational instability modified by buoyancy should be the main destabilizing factor in regions with sufficiently strong magnetic fields and/or high conductivity $\left(\Lambda>a_{\mathrm{e}}\right)$. For a magnetic field amplitude of 1 Gauss the transition between both the regions happens at approximately $1 \mathrm{AU}$.
\end{abstract}

Key words. accretion: accretion disks - magnetohydrodynamics (MHD) - instabilities - turbulence - stars: formation

\section{Introduction}

Accretion disk models require sufficiently strong turbulence to enhance the efficiency of angular momentum transport. Generally, the origin of turbulence can be attributed to various hydrodynamic and hydromagnetic instabilities that can arise in differentially rotating non-uniform gaseous disks.

It is often assumed that turbulence in accretion disks can be generated by magnetorotational instability (MRI) since the necessary condition of instability, $\partial \Omega / \partial R<0$ (i.e. a decrease of the angular velocity with cylindrical radius) is always fulfilled (Velikhov 1959; Kurzweg 1963; Balbus \& Hawley 1991; Kaisig et al. 1992; Kumar et al. 1994; Zhang et al. 1994). Apart from short-wavelength perturbations, the global modes with a length scale comparable to the disk height can be subject to the instability (Curry et al. 1994; Curry \& Pudritz 1995). The magnetic shear instability can be completely suppressed by a sufficiently strong magnetic field (see Urpin 1996; Kitchatinov \& Rüdiger 1997). Simulations of MRI in disks (Hawley et al. 1995; Matsumoto \& Tajima 1995; Brandenburg et al. 1995; Torkelsson et al. 1996; Arlt \& Rüdiger 2001) show that the turbulence generated strongly enhances angular momentum transport.

It is unlikely that the magnetic shear instability is the only instability that operates in the complex bodies that constitute astrophysical disks. A detailed analysis of MHD modes in stratified magnetic accretion disks demonstrates a much wider variety of instabilities than previously realized
(Keppens et al. 2002). Therefore, the current view of the origin of turbulence is likely highly simplified. Even a pure hydrodynamic origin of turbulence cannot be excluded (see Urpin 2003; Dubrulle et al. 2005).

The magnetic Reynolds number is likely not very large in cold and dense protostellar disks because of a low electrical conductivity, and the magnetic field cannot be treated as "frozen" into the gas (Gammie 1996). The effect of Ohmic dissipation on the magnetic shear instability has been considered in the linear (Jin 1996; Rüdiger \& Kitchatinov 2005) and nonlinear regimes (Sano et al. 1998; Drecker et al. 2000). As it was first pointed out by Wardle (1999), however, poorly conducting protostellar disks can be strongly magnetized if electrons are the main charge carriers. Therefore, the electron transport must be anisotropic with substantially different properties along and across the magnetic field (see Spitzer 1978). If the field is sufficiently strong then the main contribution to the electric resistivity tensor is provided by the Hall component that produces the electric field perpendicular to both the magnetic field and electric current. The linear stability analysis by Wardle (1999) showed that the Hall effect can provide an additional either stabilizing or destabilizing influence depending on the direction of the magnetic field. A more general consideration of the magnetic shear instability in the presence of Hall currents has been done by Balbus \& Terquem (2001). They found that the Hall effect qualitatively changes the stability properties of disks and can lead to instability even if the angular velocity increases outward (see also Rüdiger \& Shalybkov 2004). Often, however, 
gravity has been neglected. The importance of gravity in protostellar disks is stressed in the present paper. Gravity influences the behavior of modes through buoyancy and may easily change the stability properties.

In the present paper we consider the stability properties of magnetic protostellar disks. We examine the properties of magnetohydrodynamic modes that can exist in such bodies taking into account the Hall effect, Ohmic dissipation and buoyancy. The criteria of instability are derived, and the growth rate of different modes is calculated. The paper is organized as follows. In Sect. 2, we discuss the Hall effect in the conditions of protostellar clouds. In Sect. 3, the main equations are presented, and the dispersion relation is derived that describes the behavior of short wavelength perturbations in the Boussinesq approximation. The stability criteria for different modes are discussed in Sect. 4, and the growth rate of instabilities are calculated in Sect. 5. Our results are briefly summarized in Sect. 6.

\section{Anisotropic magnetic diffusivity in protostellar disks}

The electrical conductivity is likely very low in protostellar disks because of low temperature. Therefore, the magnetic field cannot be considered as "frozen" into gas, and dissipative effects should be taken into account. However, as was first pointed out by Wardle (1999), the electron gas in protostellar disks can be magnetized despite low temperature and ionization. The effect of the magnetic field on transport properties of plasma is usually characterized by the magnetization parameter $a_{\mathrm{e}}=\omega_{B} \tau$ where $\omega_{B}=e B / m_{\mathrm{e}} c$ is the gyrofrequency of electrons and $\tau$ is their relaxation time (see, e.g., Spitzer 1978). In protostellar disks, $\tau$ is determined by the scattering of electrons on neutrals, then $\tau=1 / n\langle\sigma v\rangle$ where $\langle\sigma v\rangle$ is the average product of the cross-section and velocity and $n$ is the number density of neutrals. Using the fitting expression for $\langle\sigma v\rangle$ obtained by Draine et al. (1983), we can estimate the magnetization parameter as

$a_{\mathrm{e}} \approx 21 B n_{14}^{-1} T_{2}^{-1 / 2}$,

where the magnetic field $B$ is measured in Gauss, $n_{14}=$ $n / 10^{14} \mathrm{~cm}^{-3}$ and $T_{2}=T / 100 \mathrm{~K}$ with $T$ the temperature. If $a_{\mathrm{e}}>1$, i.e.

$B>0.048 n_{14} \sqrt{T_{2}}$ Gauss

(see Fig. 1), then the electron transport is anisotropic and the magnetic diffusivity is represented by a tensor. For more details concerning the generalized Ohm's law in weakly ionized plasma we refer to the paper by Shalybkov \& Urpin (1995) where this law has been considered using the relaxation time approximation for three component plasma with ions and neutrals of the same mass. In a weakly ionized plasma of protostellar disks, the difference between components of the magnetic diffusivity parallel and perpendicular to the magnetic field is likely small (see, e.g., Balbus \& Terquem 2001), and the Hall component is given by

$a_{\mathrm{e}} \eta=\frac{c B}{4 \pi e n_{\mathrm{e}}}=\beta B$

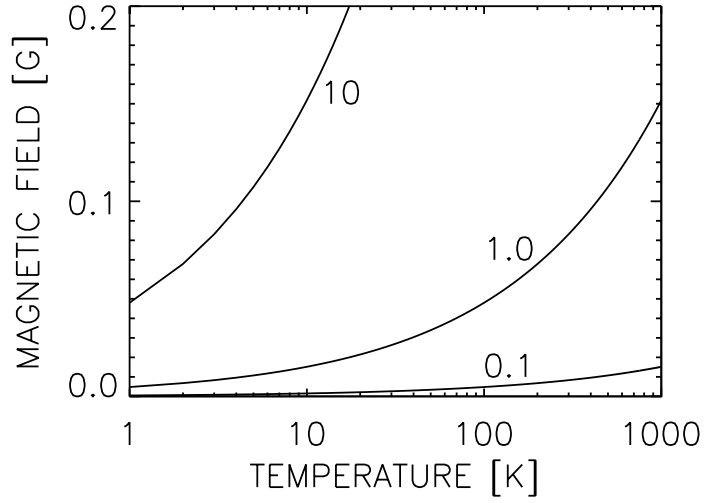

Fig. 1. The condition $a_{\mathrm{e}}=1$ where the lines are marked with the values of the number density $n_{14}$. For magnetic fields above the lines, the Hall effect dominates the Ohmic dissipation.

where $\eta=c^{2} m_{\mathrm{e}} / 4 \pi e^{2} n_{\mathrm{e}} \tau$ is the magnetic diffusivity at $B=0$; $m_{\mathrm{e}}$ and $n_{\mathrm{e}}$ are the mass and number density of electrons. Using the fit for $\langle\sigma v\rangle$ (Draine et al. 1983), we obtain

$\eta=2.34 \times 10^{3} f^{-1} T_{2}^{1 / 2} \mathrm{~cm}^{2} \mathrm{~s}^{-1}$,

where $f=n_{\mathrm{e}} / n$ is the ionization fraction.

Then, the induction equation in protostellar disks is

$\frac{\partial \boldsymbol{B}}{\partial t}=\operatorname{rot}(\boldsymbol{U} \times \boldsymbol{B})+\eta \Delta \boldsymbol{B}-\beta \operatorname{rot}(\operatorname{rot} \boldsymbol{B} \times \boldsymbol{B})$

where $\boldsymbol{U}$ is the velocity and $\beta=c / 4 \pi e n_{\mathrm{e}}$. The last term on the r.h.s. represents the Hall effect, and the numerical evaluation done by Wardle \& $\mathrm{Ng}$ (1999) indicates that this term can be of importance in some regions of protostellar disks. In Eq. (5), we neglect non-uniformity of the magnetic diffusivity and electron number density.

\section{Basic equations and the dispersion relation}

Consider stability of a magnetized axisymmetric protostellar disk of a finite vertical extent. The unperturbed angular velocity can generally depend on both $R$ and $z$, so $\Omega=\Omega(R, z)$, where $(R, \phi, z)$ are cylindrical coordinates. The magnetic field, $\boldsymbol{B}=\left(B_{R}, B_{\phi}, B_{z}\right)$, is assumed to be weak in the sense that the Alfvén speed, $V_{\mathrm{A}}$, is small compared to the sound speed, $c_{\mathrm{s}}$. This enables us to employ the Boussinesq approximation for a consideration of slowly varying modes.

In the unperturbed state, the disk is assumed to be in hydrostatic equilibrium in the $R$ - and $z$-directions,

$\frac{\nabla p}{\rho}=\boldsymbol{G}+\frac{1}{4 \pi \rho} \operatorname{rot} \boldsymbol{B} \times \boldsymbol{B}, \quad \boldsymbol{G}=\boldsymbol{g}+\Omega^{2} \boldsymbol{R}$,

where $\boldsymbol{g}$ is the gravity. If $c_{\mathrm{S}}>V_{\mathrm{A}}$, the unperturbed Lorentz force is small compared to the pressure force, thus the disk structure is mainly determined by the balance between gravity, centrifugal force and pressure.

We consider axisymmetric short wavelength perturbations with the space-time dependence $\exp (\gamma t-\mathrm{i} \boldsymbol{k} \cdot \boldsymbol{x})$ where $\boldsymbol{k}=$ $\left(k_{R}, 0, k_{z}\right)$ is the wave vector. Small perturbations will be indicated by subscript 1 , whilst unperturbed quantities will have no subscript, except for indicating vector components when 
necessary. The linearized momentum and continuity equations governing the behavior of such perturbations in the Boussinesq approximation are

$$
\begin{aligned}
\gamma \boldsymbol{U}^{\prime}+ & 2 \boldsymbol{\Omega} \times \boldsymbol{U}^{\prime}+\hat{\boldsymbol{e}}_{\phi} R\left(\boldsymbol{U}^{\prime} \cdot \nabla\right) \Omega=\frac{\mathrm{i} \boldsymbol{k} P^{\prime}}{\rho}-\alpha \boldsymbol{G} T^{\prime} \\
& +\frac{\mathrm{i}}{4 \pi \rho}\left[\left(\boldsymbol{B} \cdot \boldsymbol{B}^{\prime}\right) \boldsymbol{k}-(\boldsymbol{k} \cdot \boldsymbol{B}) \boldsymbol{B}^{\prime}\right], \quad \boldsymbol{k} \cdot \boldsymbol{U}^{\prime}=0,
\end{aligned}
$$

where $\boldsymbol{U}^{\prime}, \boldsymbol{B}^{\prime}, P^{\prime}$ and $T^{\prime}$ are perturbations of the hydrodynamic velocity, magnetic field, pressure and temperature, respectively; $\alpha=-(\partial \ln \rho / \partial T)_{P}$ is the thermal expansion coefficient and $\hat{\boldsymbol{e}}_{\phi}$ is the unit vector in the azimuthal direction. In Eq. (7), it is assumed that the density perturbation in the buoyancy force is determined by the temperature perturbation alone in accordance with the main idea of the Boussinesq approximation, $\rho^{\prime}=-\rho \alpha T^{\prime}$.

Since the thermal conductivity of protostellar clouds is low because of low temperature $\left(T \sim 10-10^{3} \mathrm{~K}\right)$, we adopt the adiabatic equation to describe the evolution of temperature perturbations,

$\gamma T^{\prime}+\boldsymbol{U}^{\prime} \cdot(\Delta \nabla T)=0$,

where $(\Delta \nabla T)=\nabla T-\nabla_{\mathrm{ad}} T$ is the difference between the actual and adiabatic temperature gradients.

The linearized induction equation and the divergence-free condition are

$$
\begin{aligned}
\left(\gamma+\omega_{\eta}\right) \boldsymbol{B}^{\prime}=- & \mathrm{i} \boldsymbol{U}^{\prime}(\boldsymbol{k} \cdot \boldsymbol{B})+\boldsymbol{R e}_{\phi}\left(\boldsymbol{B}^{\prime} \cdot \nabla \Omega\right) \\
& +\beta(\boldsymbol{k} \cdot \boldsymbol{B}) \boldsymbol{k} \times \boldsymbol{B}^{\prime}, \quad \boldsymbol{k} \cdot \boldsymbol{B}^{\prime}=0,
\end{aligned}
$$

where $\omega_{\eta}=\eta k^{2}$ is the inverse timescale of Ohmic dissipation.

The dispersion equation from the Eqs. (7)-(9) is

$$
\gamma^{5}+a_{4} \gamma^{4}+a_{3} \gamma^{3}+a_{2} \gamma^{2}+a_{1} \gamma+a_{0}=0,
$$

where $a_{4}=2 \omega_{\eta}$,

$a_{3}=\omega_{\eta}^{2}+\omega_{\mathrm{H}}\left(\omega_{\mathrm{H}}+\omega_{\mathrm{sh}}\right)+2 \omega_{\mathrm{A}}^{2}+\omega_{\mathrm{g}}^{2}+Q^{2}$,

$a_{2}=2 \omega_{\eta}\left(\omega_{\mathrm{g}}^{2}+\omega_{\mathrm{A}}^{2}+Q^{2}\right)$,

$a_{1}=\left[\omega_{\eta}^{2}+\omega_{\mathrm{H}}\left(\omega_{\mathrm{H}}+\omega_{\mathrm{sh}}\right)\right]\left(\omega_{\mathrm{g}}^{2}+Q^{2}\right)$

$$
+\omega_{\mathrm{A}}^{2}\left[\omega_{\mathrm{g}}^{2}+\omega_{\mathrm{A}}^{2}+\omega_{\mathrm{H}} \omega_{\mathrm{sh}}+2 \Omega \frac{k_{z}}{k}\left(2 \omega_{\mathrm{H}}+\omega_{\mathrm{sh}}\right)\right],
$$

$a_{0}=\omega_{\eta} \omega_{\mathrm{A}}^{2} \omega_{\mathrm{g}}^{2}$ and

$Q^{2}=4 \Omega^{2} \frac{k_{z}^{2}}{k^{2}}+2 \Omega R \frac{k_{z}}{k^{2}}\left(k_{z} \frac{\partial \Omega}{\partial R}-k_{R} \frac{\partial \Omega}{\partial z}\right)$,

$\omega_{\mathrm{g}}^{2}=-\alpha \Delta \nabla T \cdot\left[\boldsymbol{G}-\frac{\boldsymbol{k}}{k^{2}}(\boldsymbol{k} \cdot \boldsymbol{G})\right]$,

$\omega_{\mathrm{H}}=\beta k(\boldsymbol{k} \cdot \boldsymbol{B}), \quad \omega_{\mathrm{A}}=(\boldsymbol{k} \cdot \boldsymbol{B}) / \sqrt{4 \pi \rho}$

$\omega_{\mathrm{sh}}=\frac{R}{k}\left(k_{z} \frac{\partial \Omega}{\partial R}-k_{R} \frac{\partial \Omega}{\partial z}\right)$,

$\omega_{\mathrm{g}}$ is the frequency of buoyancy waves; $\omega_{\mathrm{A}}$ is the Alfvén frequency, $Q^{2}$ represents the effects associated with differential rotation, $\omega_{\mathrm{H}}$ and $\omega_{\mathrm{sh}}$ are the characteristic frequencies of the Hall- and shear-driven processes.
If gravity is neglected $\left(\omega_{\mathrm{g}}=0\right)$ then we recover the dispersion equation derived by Balbus \& Terquem (2001)

$\gamma^{4}+b_{3} \gamma^{3}+b_{2} \gamma^{2}+b_{1} \gamma+b_{0}=0$,

where $b_{3}=2 \omega_{\eta}$,

$$
\begin{aligned}
b_{2}= & \omega_{\eta}^{2}+\omega_{\mathrm{H}}\left(\omega_{\mathrm{H}}+\omega_{\mathrm{sh}}\right)+2 \omega_{\mathrm{A}}^{2}+Q^{2}, \\
b_{1}= & 2 \omega_{\eta}\left(\omega_{\mathrm{A}}^{2}+Q^{2}\right), \\
b_{0}= & \omega_{\eta}^{2} Q^{2}+\left[\omega_{\mathrm{A}}^{2}+2 \Omega \frac{k_{z}}{k}\left(\omega_{\mathrm{H}}+\omega_{\mathrm{sh}}\right)\right] \\
& \times\left[\omega_{\mathrm{A}}^{2}+\omega_{\mathrm{H}}\left(2 \Omega \frac{k_{z}}{k}+\omega_{\mathrm{sh}}\right)\right] .
\end{aligned}
$$

Assuming that instability arises if $b_{0}<0$, Balbus \& Terquem (2001) argued that the Hall effect can destabilize any differential rotation.

\section{Criteria of instability of protostellar disks}

Equation (10) describes five low-frequency modes that exist in protostellar disks. The Hurwitz theorem states that at least one of the roots of Eq. (10) has a positive real part (unstable mode) if one of the following inequalities

$$
\begin{aligned}
& a_{4}<0, \quad a_{0}<0, \\
& A_{1} \equiv a_{4} a_{3}-a_{2}<0, \\
& A_{2} \equiv a_{2}\left(a_{4} a_{3}-a_{2}\right)-a_{4}\left(a_{4} a_{1}-a_{0}\right)<0, \\
& A_{3} \equiv\left(a_{4} a_{1}-a_{0}\right)\left[a_{2}\left(a_{4} a_{3}-a_{2}\right)-a_{4}\left(a_{4} a_{1}-a_{0}\right)\right] \\
& \quad-a_{0}\left(a_{4} a_{3}-a_{2}\right)^{2}<0
\end{aligned}
$$

is fulfilled (see Aleksandrov et al. 1985). Since $\omega_{\eta}$ is a positive defined quantity, the first condition $\left(a_{4}<0\right)$ will never apply, and only the four remaining conditions determine the instability in disks. Note that there is no one-to-one correspondence between the criteria and modes. For instance, few modes can generally be unstable if only one criteria (14) is fulfilled and, on the contrary, it is also possible that only one mode is unstable if few criteria are satisfied. The conditions (14) determine only the region where Eq. (10) has unstable solutions.

\subsection{The condition $a_{0}<0$}

Since $\omega_{\mathrm{A}}^{2}>0$, the condition $a_{0}<0$ is equivalent to

$\omega_{\mathrm{g}}^{2}<0$

or

$k^{2}(\boldsymbol{G} \cdot \Delta \nabla T)-(\boldsymbol{G} \cdot \boldsymbol{k})(\boldsymbol{k} \cdot \Delta \nabla T)>0$.

Generally, the standard convective instability arises if $\nabla T \| \boldsymbol{g}$ and $\omega_{\mathrm{g}}^{2}<0$. However, $\omega_{\mathrm{g}}^{2}$ may also be negative if the temperature gradient is subadiabatic but $\Delta \nabla T$ is not parallel to the "effective gravity", $\boldsymbol{G}$ (Knobloch \& Spruit 1986; Urpin \& Brandenburg 1998; Balbus 2000). This obliqueness can be caused, in principle, either by the dependence of $\Omega$ on $z$ or by radiative heat transport in the radial direction. Introducing the angle $\psi$ between the vectors $\boldsymbol{G}$ and $\boldsymbol{k}$ and representing $\Delta \nabla T$ as 
a sum of components parallel and perpendicular to $G, \Delta \nabla T=$ $(\Delta \nabla T)_{\|}+(\Delta \nabla T)_{\perp}$, the inequality (16) can be rewritten in the form

$G(\Delta \nabla T)_{\|}\left[\sin ^{2} \psi-\sin \psi \cos \psi(\Delta \nabla T)_{\perp} /(\Delta \nabla T)_{\|}\right]>0$.

If stratification is stable according to the standard Schwarzschild criterion of convection, $G(\Delta \nabla T)_{\|}<0$, then the instability arises at

$\sin ^{2} \psi-\sin \psi \cos \psi \frac{(\Delta \nabla T)_{\perp}}{(\Delta \nabla T)_{\|}}<0$.

This condition can be fulfilled due to the obliqueness of $\boldsymbol{G}$ and $\Delta \nabla T$ for perturbations with a small (but nonzero) angle $\psi$. Estimating $(\Delta \nabla T)_{\perp}$ as $(z / R)(\Delta \nabla T)_{\|}$in protostellar disks, we obtain that $\omega_{\mathrm{g}}^{2}$ for unstable perturbations is small,

$\omega_{\mathrm{g}}^{2} \sim-\Omega^{2}(H / R)^{2}$.

The baroclinic convection is therefore probably relatively slow.

\subsection{The condition $A_{1}<0$}

This condition can be rewritten as

$\omega_{\mathrm{A}}^{2}+\omega_{\eta}^{2}+\omega_{\mathrm{H}}^{2}+\omega_{\mathrm{H}} \omega_{\mathrm{sh}}<0$,

or, substituting the frequencies,

$\beta R(\boldsymbol{k} \cdot \boldsymbol{B})\left(k_{z} \frac{\partial \Omega}{\partial R}-k_{R} \frac{\partial \Omega}{\partial z}\right)<-\omega_{\mathrm{A}}^{2}-\omega_{\eta}^{2}-\beta^{2} k^{2}(\boldsymbol{k} \cdot \boldsymbol{B})^{2}$.

The inequality (20) is the only criterion (14) that does not depend on gravity. Therefore, exactly the same condition can also be obtained by applying the Hurwitz theorem to the dispersion Eq. (81) of the paper by Balbus \& Terquem (2001) where the effect of gravity is neglected.

Note that even if gravity is negligible, the criterion (20) is not equivalent to the condition (84) of Balbus \& Terquem (2001). This is seen, for example, in the particular case of astrophysical interest when dissipation is weak $\left(\omega_{\eta} \rightarrow 0\right)$ and $\Omega$ is decreasing with $R$. In our notations, the condition of instability derived by Balbus \& Terquem (2001) for this case (Eq. (85) of their paper) can be rewritten as

$\beta R(\boldsymbol{k} \cdot \boldsymbol{B}) k_{z} \frac{\partial \Omega}{\partial R}<-\xi \omega_{\mathrm{A}}^{2}-\beta^{2} k^{2}(\boldsymbol{k} \cdot \boldsymbol{B})^{2}$

where

$\xi=\frac{k^{2}}{k_{z}^{2}} \frac{\beta k_{z}(\boldsymbol{k} \cdot \boldsymbol{B})}{2 \Omega}$.

Even if one neglects the vertical shear, the inequality (22) differs from (21). For instance, if $\xi>1$ then the strength of the magnetic field that suppresses the instability is lower for condition (22) and, hence, the range of magnetic fields that allow for instability is wider for condition (21).

Condition (21) describes the instability that appears due to the combined influence of shear and the Hall effect. This instability differs from the magnetic shear instability because the only term that can provide a destabilizing influence is proportional to the Hall frequency and shear stresses, and this term vanishes if $\omega_{\mathrm{H}} \rightarrow 0$. To satisfy the inequality (21) the sign of the left hand side should be negative since all three terms on the right hand side are negative. Obviously, for any dependence of $\Omega$ on $R$ and $z$ and for any direction of $\boldsymbol{B}$, there exist the wave vectors that satisfy the inequality

$(\boldsymbol{k} \cdot \boldsymbol{B})\left(k_{z} \frac{\partial \Omega}{\partial R}-k_{R} \frac{\partial \Omega}{\partial z}\right)<0$

and make the left hand side of Eq. (21) negative. Therefore, any differential rotation can generally be unstable if the Hall frequency is sufficiently large. Note that the condition of instability (24) cannot be satisfied for negative shear if $\boldsymbol{B}$ is antiparallel to $\boldsymbol{\Omega}$.

Compare the characteristic frequencies in Eq. (20). If the Hall parameter is large, $a_{\mathrm{e}} \gg 1$, then the "Ohmic frequency" is negligible in Eq. (20). The characteristic value of the Hall frequency, $\omega_{\mathrm{H}}$, is

$\omega_{\mathrm{H}} \sim 2 \times 10^{-4} B n_{\mathrm{e} 2}^{-1} \lambda_{11}^{-2} \mathrm{~s}^{-1}$,

where $\lambda=2 \pi / k$ is the wavelength, and $\lambda_{11}=\lambda / 10^{11} \mathrm{~cm}$; $n_{\mathrm{e} 2}=n_{\mathrm{e}} / 100 \mathrm{~cm}^{-3}$. Assuming that plasma is weakly ionized and $n \gg n_{\mathrm{e}}$, we can estimate

$\left|\frac{\omega_{\mathrm{H}}}{\omega_{\mathrm{A}}}\right| \approx \frac{c k}{\omega_{\mathrm{p}}} \sqrt{\frac{m_{\mathrm{p}} n}{m_{\mathrm{e}} n_{\mathrm{e}}}} \approx 1.4 \times 10^{-4} \lambda_{11}^{-1} n_{\mathrm{e} 2}^{-1 / 2} f^{-1 / 2}$

where $\omega_{\mathrm{p}}=\left(4 \pi e^{2} n_{\mathrm{e}} / m_{\mathrm{e}}\right)^{1 / 2}$ is the plasma frequency, $m_{\mathrm{e}}$ and $m_{\mathrm{p}}$ are the electron and proton mass, respectively. Since $f$ can be very small in a large fraction of the disk volume $(f \sim$ $\left.10^{-(11-13)}\right)$, most likely $\left|\omega_{\mathrm{H}}\right|>\left|\omega_{\mathrm{A}}\right|$. In this case, the Alfvén frequency is small in (20). Then, the condition of instability is

$\omega_{\mathrm{H}} \omega_{\mathrm{sh}}<-\omega_{\mathrm{H}}^{2}$

Assuming $|\partial \Omega / \partial R| \sim \Omega / R$, we can estimate the magnetic field satisfying the condition (27) as

$B<\frac{\Omega}{\beta k^{2}} \simeq 10^{-1} P_{\mathrm{yr}}^{-1} n_{\mathrm{e} 2} \lambda_{12}^{2}$ Gauss,

where $P_{\mathrm{yr}}$ is the rotation period in years. On the other hand, the condition $a_{\mathrm{e}}>1$ yields

$B>0.048 n_{14} T_{2}^{1 / 2}$ Gauss.

The conditions (28) and (29) are consistent only if

$P_{\mathrm{yr}}<2.2 f_{-12} \lambda_{12}^{2} T_{2}^{-1 / 2}$

where $f_{-12}=f / 10^{-12}$. For $P=1 \mathrm{yr}$ it follows $\lambda>10^{12} \mathrm{~cm}$ $(\simeq 0.1 \mathrm{AU})$. The corresponding magnetic amplitude is larger than 0.05 Gauss. Note that the Hall instability (27) only exists for relatively thick disks under the influence of rather strong magnetic fields. Instabilities associated with the criterion (20) do not occur if the conductivity in protoplanetary disks is very low and either $\omega_{\eta}>\Omega$ or $\omega_{\mathrm{H}}>\Omega$. 


\subsection{The condition $A_{2}<0$}

The condition $A_{2}<0$ can be rewritten as

$\omega_{\mathrm{g}}^{2}+2 \omega_{\eta}^{2}+2\left(2 \Omega \frac{k_{z}}{k}-\omega_{\mathrm{H}}\right)^{2}<0$.

All terms on the left hand side are positive except $\omega_{\mathrm{g}}^{2}$ and, hence, the criterion (31) can be satisfied only if $\omega_{\mathrm{g}}^{2}<0$. However, this criterion requires even larger negative $\omega_{\mathrm{g}}^{2}$ compared to (15) so that no new instability arises from $A_{2}<0$.

\subsection{The condition $A_{3}<0$}

The condition $A_{3}<0$ generalizes the criterion obtained by Balbus \& Terquem (2001) for the protostellar disk with no gravity. If we assume $\boldsymbol{g}=0$ (and, hence, $\omega_{\mathrm{g}}^{2}=a_{0}=0$ ) then $A_{3}=a_{4} a_{1} A_{2}$. Since $a_{4}$ is always positive and $A_{2}$ is positive at $\boldsymbol{g}=0$ (see Eq. (31)), the criterion $A_{3}<0$ reduces to

$\left.a_{1}\right|_{g=0} \equiv b_{0}<0$,

i.e. the condition of Balbus \& Terquem (2001).

The condition $A_{3}<0$ is equivalent to

$$
\begin{aligned}
D \equiv & b_{0}+q\left\{( \omega _ { \eta } ^ { 2 } + \omega _ { \mathrm { H } } ^ { 2 } + \omega _ { \mathrm { H } } \omega _ { \mathrm { sh } } ) \left[\omega_{\mathrm{g}}^{2}+Q^{2}-\omega_{\mathrm{A}}^{2}\right.\right. \\
& \left.+\omega_{\eta}^{2}+2\left(2 \Omega \frac{k_{z}}{k}-\omega_{\mathrm{H}}\right)^{2}-\omega_{\mathrm{H}}^{2}-\omega_{\mathrm{H}} \omega_{\mathrm{sh}}\right] \\
& \left.+\omega_{\mathrm{A}}^{2}\left(\frac{1}{2} \omega_{\mathrm{g}}^{2}+Q^{2}\right)\right\}<0,
\end{aligned}
$$

where

$q=\frac{1}{2} \frac{\omega_{\mathrm{g}}^{2}}{\omega_{\eta}^{2}+\left(2 \Omega \frac{k_{z}}{k}-\omega_{\mathrm{H}}\right)^{2}}$.

Gravity can provide either positive or negative contributions to the LHS of Eq. (33) and, hence, can be either a stabilizing or destabilizing factor. The condition (33) is rather cumbersome, so that only the particular cases of astrophysical interest are considered.

Generally, $q$ can be of the order of 1 in protostellar disks, and both terms in Eq. (33) are of importance. We assume that the Hall effect dominates the Ohmic dissipation, i.e. $\omega_{\mathrm{H}}>\omega_{\eta}$ with

$\omega_{\eta} \approx 9.2 \times 10^{-6} f_{-12}^{-1} T_{2}^{1 / 2} \lambda_{11}^{-2} \mathrm{~s}^{-1}$.

The stability properties prove to be sensitive to the relation between the Hall frequency, $\omega_{\mathrm{H}}$, and the angular velocity, $\Omega$. Consider the instability in the disk with a relatively high conductivity when $\Omega \gg \omega_{\mathrm{H}}$. Then (33) reads

$$
\begin{aligned}
& \omega_{\mathrm{H}}\left(\omega_{\mathrm{H}}+\omega_{\mathrm{sh}}\right)\left[(1+q)\left(\omega_{\mathrm{g}}^{2}+Q^{2}\right)-q \omega_{\mathrm{A}}^{2}\right]+\omega_{\mathrm{A}}^{4} \\
& +\omega_{\mathrm{A}}^{2}\left[\omega_{\mathrm{H}}\left(2 \Omega \frac{k_{z}}{k}+\omega_{\mathrm{sh}}\right)+2 \Omega \frac{k_{z}}{k}\left(\omega_{\mathrm{H}}+\omega_{\mathrm{sh}}\right)\right] \\
& +q \omega_{\mathrm{A}}^{2}\left(\frac{1}{2} \omega_{\mathrm{g}}^{2}+Q^{2}\right)<0 .
\end{aligned}
$$

We consider both the cases of strong Hall effect $\left(\omega_{\mathrm{H}}>\omega_{\mathrm{A}}\right)$ and "weak" Hall effect $\left(\omega_{\mathrm{H}}<\omega_{\mathrm{A}}\right)$. If $\omega_{\mathrm{H}}>\omega_{\mathrm{A}}$ then (36) yields

$\omega_{\mathrm{H}}\left(\omega_{\mathrm{H}}+\omega_{\mathrm{sh}}\right)(1+q)\left(\omega_{\mathrm{g}}^{2}+Q^{2}\right)<0$.

In a convectively stable disk with $\omega_{\mathrm{g}}^{2}>0$ and $Q^{2}>0$, the shear-driven instability arises if

$\omega_{\mathrm{H}}\left(\omega_{\mathrm{H}}+\omega_{\mathrm{sh}}\right)<0$.

A strong shear is thus required. The inequality $\left|\omega_{\mathrm{sh}}\right|>\left|\omega_{\mathrm{H}}\right|$ is the necessary condition for instabilities caused by the combined influence of shear and the Hall effect but, as mentioned, this inequality can be fulfilled only if the magnetic field is relatively strong. The sign of $\omega_{\text {sh }}$ plays no crucial role in Eq. (38) so that instability can appear for any $\partial \Omega / \partial R$ if Eq. (27) is fulfilled.

Consider now the instability condition (36) for the "weak" Hall effect $\omega_{\mathrm{H}}<\omega_{\mathrm{A}}$. In protostellar disks, the Alfvén frequency can be larger or smaller than the angular velocity, $\Omega$. If $\omega_{\mathrm{A}}>\Omega$ then Eq. (36) cannot easily be fulfilled. The needed field would be even stronger than 1 Gauss. In the more realistic case of fast rotation $\Omega>\omega_{\mathrm{A}}$ (then also $\Omega>\omega_{\mathrm{H}}$ ) we must distinguish between the two possibilities, $\omega_{\mathrm{A}}>\sqrt{\omega_{\mathrm{H}} \Omega}$ and $\sqrt{\omega_{\mathrm{H}} \Omega}>\omega_{\mathrm{A}}$. If $\omega_{\mathrm{A}}>\sqrt{\omega_{\mathrm{H}} \Omega}$ then

$(1+q)\left(\omega_{\mathrm{g}}^{2}+4 \Omega \frac{k_{z}}{k} \omega_{\mathrm{sh}}\right)<0$.

In a convectively stable disk, the instability appears if

$4 \Omega \frac{k_{z}}{k} \omega_{\mathrm{sh}}+\omega_{\mathrm{g}}^{2}<0$

At $\omega_{\mathrm{g}} \sim \Omega \gg \omega_{\mathrm{A}}$, this condition is highly restrictive. The Hall effect only plays a modifying role.

If $\sqrt{\omega_{\mathrm{H}} \Omega}>\omega_{\mathrm{A}}$ then the instability criterion (37) for the Hall-driven shear instability is recovered. Hence, if the rotation is fast enough then also for a "weak" Hall effect the sheardriven Hall instability can exist.

If the ionization fraction in protostellar disks is so low (Igea \& Glassgold 1999) that the magnetic Reynolds number is smaller than unity then the Ohmic dissipation dominates $\left(\omega_{\eta}>\Omega\right)$ and, hence, $q \approx \omega_{\mathrm{g}}^{2} / 2\left(\omega_{\eta}^{2}+\omega_{\mathrm{H}}^{2}\right)$ in Eq. (33). Assuming the Hall parameter as large $\left(\omega_{\mathrm{H}}>\omega_{\eta}\right)$, we have from Eq. (33)

$D \approx b_{0}+\frac{1}{2} \omega_{\mathrm{H}}^{2} \omega_{\mathrm{g}}^{2} \approx \frac{1}{2}\left(\omega_{\mathrm{g}}^{2}+2 Q^{2}\right)$.

Obviously, the condition $D<0$ cannot be satisfied and instabilities associated with this criterion do not occur (Balbus \& Terquem 2001).

\section{The growth rate of instability}

From the dispersion relation (10) relatively simple expressions for the roots can be obtained in some particular cases of astrophysical interest. Consider initially Eq. (10) in the case when the Ohmic dissipative frequency, $\omega_{\eta}$, is small compared to other characteristic frequencies. Then in terms of the zeroth order in $\omega_{\eta}$ four roots corresponding to rapidly varying modes satisfy

$\gamma^{4}+c_{2} \gamma^{2}+c_{0}=0$ 
where $c_{0}=\omega_{\mathrm{A}}^{2}\left(\omega_{\mathrm{g}}^{2}+2 \Omega \frac{k_{z}}{k} \omega_{\mathrm{sh}}\right)+\omega_{\mathrm{H}}\left(\omega_{\mathrm{H}}+\omega_{\mathrm{sh}}\right)\left(\omega_{\mathrm{g}}^{2}+Q^{2}\right)$ and $c_{2} \approx \omega_{\mathrm{g}}^{2}+Q^{2}$. For $\boldsymbol{B}=0$ the quantity $c_{0}$ vanishes so that $\gamma^{2}=-c_{2}$ solves Eq. (42). The corresponding instability condition $\omega_{\mathrm{g}}^{2}+Q^{2}<0$ can easily be written in the form of the Solberg-Høiland criterion. The fifth mode describes a secular instability and varies on a long timescale proportional to $\omega_{\eta}^{-1}$. In terms of the lowest order in $\omega_{\eta}$

$\gamma_{5} \approx-\frac{a_{0}}{a_{1}} \approx-\frac{1}{c_{0}} \omega_{\eta} \omega_{\mathrm{A}}^{2} \omega_{\mathrm{g}}^{2}$.

The roots of Eq. (42) are

$\gamma^{2}=-\frac{c_{2}}{2} \pm \sqrt{\frac{c_{2}^{2}}{4}-c_{0}}$.

Note that these roots only hold in the lowest order in $\omega_{\eta}$. Generally, small corrections, $\Delta \gamma$, proportional to $\omega_{\eta}$ can be calculated from the dispersion equation (10).

If $\Omega>\max \left(\omega_{\mathrm{H}}, \omega_{\mathrm{A}}\right)$, then $c_{2}^{2} \gg 4 c_{0}$ and

$\gamma_{1,2}^{2} \approx-\frac{c_{0}}{c_{2}}, \quad \gamma_{3,4}^{2} \approx-c_{2}$.

The modes 1 and 2 are relevant to the Hall-driven instability whereas the modes 3 and 4 describe convection and are stable in convectively stable disks. One of the modes $\gamma_{1,2}$ is unstable if $c_{0}<0$. Note that the secular mode (43) also is unstable under this condition, and there exist two qualitatively different instabilities represented by the same criterion.

For $\sqrt{\omega_{\mathrm{H}} \Omega}>\omega_{\mathrm{A}}$ we obtain from Eq. (45)

$\gamma_{1,2}^{2} \approx-\omega_{\mathrm{H}}\left(\omega_{\mathrm{H}}+\omega_{\mathrm{sh}}\right)$.

If the condition (38) is fulfilled then one of these modes is unstable. Since $\omega_{\mathrm{sh}} \sim \Omega$, we can estimate

$\left|\frac{\gamma_{1,2}}{\Omega}\right| \sim \sqrt{\frac{\omega_{\mathrm{H}}}{\Omega}} \sim 31 B^{1 / 2} n_{\mathrm{e} 2}^{-1 / 2} \lambda_{11}^{-1} P_{\mathrm{yr}}^{1 / 2}$.

If $\Omega>\omega_{\mathrm{A}}>\sqrt{\omega_{\mathrm{H}} \Omega}$ then we have from Eq. (45)

$\gamma_{1,2}^{2} \approx-\frac{\omega_{\mathrm{A}}^{2}}{\omega_{\mathrm{g}}^{2}+Q^{2}}\left(\omega_{\mathrm{g}}^{2}+2 \Omega \frac{k_{z}}{k} \omega_{\mathrm{sh}}\right)$.

This is the well-known dispersion equation for magnetic sheardriven modes in the limit $\omega_{\mathrm{g}} \gg \omega_{\mathrm{A}}$ (Balbus 1995; Urpin 1996), and one of these modes is unstable if

$2 \Omega \frac{k_{z}}{k} \omega_{\mathrm{sh}}<-\omega_{\mathrm{g}}^{2}$.

The growth rate is of the order of $\omega_{\mathrm{A}}$. Note that condition (49) differs from the more general condition (40) that predicts instability also within the range

$-\omega_{\mathrm{g}}^{2}<2 \Omega \frac{k_{z}}{k} \omega_{\mathrm{sh}}<-\frac{1}{2} \omega_{\mathrm{g}}^{2}$.

The difference originates from the approximate character of Eq. (45) that describes $\gamma_{1,2}^{2}$ neglecting terms proportional to $\omega_{\eta}$. If the inequality (49) is not fulfilled, we have $c_{0}>0$, and the roots $\gamma_{1}$ and $\gamma_{2}$ become imaginary. Therefore, their stability at $c_{0}>0$ is determined by small real corrections $\propto \omega_{\eta}$. These corrections can be calculated by making use of the standard perturbation procedure. For the modes 1 and 2 the corrections are

$\Delta \gamma_{1,2} \approx \frac{1}{2 c_{0}}\left(a_{0}-2 a_{4} c_{0}\right)=-\frac{\omega_{\eta} \omega_{\mathrm{A}}^{2}}{2 c_{0}}\left(4 \Omega \frac{k_{z}}{k} \omega_{\mathrm{sh}}+\omega_{\mathrm{g}}^{2}\right)$.

They are positive under condition (50) and lead to instability, in complete agreement with criterion (40). However, the instability caused by small resistive corrections is oscillatory and qualitatively different from the magnetic shear instability. Note that within the range (50) the secular mode (43) is stable.

Consider now the roots of Eq. (10) assuming that the dissipative "frequency", $\omega_{\eta}$, is large compared to other characteristic frequencies including $\omega_{\mathrm{H}}$. Most likely the angular velocity $\Omega$ is the largest one among the other frequencies, and the assumption $\omega_{\eta}>\Omega$ implies that

$P_{\mathrm{yr}}>2.2 \times 10^{-2} f_{-12} \lambda_{11}^{2} T_{2}^{-1 / 2}$.

Probably, such an inequality can be fulfilled in some regions of protostellar disks if ionization is low. In this case, the coefficients of Eq. (10) are given by $a_{4} \simeq 2 \omega_{\eta}, a_{3} \simeq \omega_{\eta}^{2}, a_{2} \simeq$ $a_{4}\left(\omega_{\mathrm{g}}^{2}+Q^{2}\right), a_{1} \simeq \omega_{\eta} a_{2} / 2$ and $a_{0} \simeq \omega_{\eta} \omega_{\mathrm{A}}^{2} \omega_{\mathrm{g}}^{2}$. Four roots of Eq. (10) with these coefficients have negative real parts, and only one root corresponds to an instability. In the lowest order in $\omega_{\eta}^{-1}$, this root is given by

$\gamma \approx-\frac{\omega_{\mathrm{A}}^{2}}{\omega_{\eta}} \cdot \frac{\omega_{\mathrm{g}}^{2}}{\omega_{\mathrm{g}}^{2}+Q^{2}}$.

The growth rate is positive if $\omega_{\mathrm{g}}^{2}<0$. As it was argued above (see Eq. (15)), the instability can appear if $\boldsymbol{G}$ and $\Delta \nabla T$ are not parallel. In protostellar disks, however, the growth rate of this instability is relative small,

$\gamma \sim \omega_{\mathrm{A}} \frac{\omega_{\mathrm{A}}}{\omega_{\eta}}\left(\frac{H}{R}\right)^{2}$.

This instability can exist only if the ionization is extremely low and the magnetic Reynolds number is small even for rotation.

\section{Discussion}

We have considered both the criteria and growth rates of various instabilities that can arise in protostellar disks. Some of these instabilities are relevant to the Hall effect but some others can manifest themselves even if the Hall current is negligible. All considered instabilities are influenced by the magnetic field. The strength of the field that stabilizes the flow depends generally on the conditions in a disk and the wavelength of the perturbation. The stabilizing field is determined by Eqs. (28) and (29) and it is typically relatively strong, exceeding 1 Gauss.

The type of most rapidly growing instability depends very much on the conditions in protostellar disks and can be different in different regions. The instability associated with criterion (15) seems to be most general in protostellar disks. It can occur both in magnetized $\left(a_{\mathrm{e}}>1\right)$ and non-magnetized $\left(a_{\mathrm{e}}<1\right)$ regions and requires only non-parallel $\boldsymbol{G}$ and $\Delta \nabla T$. It is noteworthy that the baroclinic instability appears only if 
dissipative processes are taken into account. The baroclinic convection also exists in non-magnetic accretion disks where the dominating dissipative process is heat transport (Urpin 2004). The growth rate of this instability is given by Eqs. (43) and (54) for small and large $\omega_{\eta}$ and depends substantially on the buoyant frequency $\omega_{\mathrm{g}}$. For the most rapidly growing perturbations this frequency is given by Eq. (43). In a thin disk we have $H \sim c_{\mathrm{s}} / \Omega$ so that

$\omega_{\mathrm{g}} \sim \frac{c_{\mathrm{s}}}{R} \sim 10^{-8} T_{2}^{1 / 2} R_{13}^{-1} \mathrm{~s}^{-1}$.

Note that $\omega_{\mathrm{g}}$ can be larger than this value by a factor $(R / H)^{1 / 2}$ for stable perturbations. The buoyant frequency is very small compared to the Keplerian angular velocity. However, the baroclinic convection can occur even in disk regions with extremely weak ionization where other instabilities are suppressed. This might be of particular importance for the so-called dead zones in protostellar disks (Igea \& Glassgold 1999; Sano \& Stone $2002 a, b)$. The dead zone is usually defined as a region where $\eta>H V_{\mathrm{A}}\left(V_{\mathrm{A}}\right.$ is the Alfvén velocity). The conductivity in this region can be very low (but still non-vanishing) and, as a result, the MRI is suppressed by the Ohmic dissipation (see, e.g., Gammie 1996). This zone ranges typically from $0.1 \mathrm{AU}$ to $\sim 5$ AU. The baroclinic convection seems to be the only instability that can operate in the dead zone since criterion (15) does not depend on the conductivity. The growth rate in the dead zone can be estimated with Eq. (53). Assuming that $H / R \sim 0.1$, we have

$\gamma \sim \frac{V_{\mathrm{A}}^{2}}{\eta}\left(\frac{H}{R}\right)^{2} \sim 3 \times 10^{-9} B^{2} n_{14}^{-1} f_{-12} T_{2}^{1 / 2} \mathrm{~s}^{-1}$.

Contrary to the criterion (15), the growth rate of the baroclinic instability depends on the conductivity which is smaller for a lower ionization fraction. For the protostellar disk model of Igea \& Glassgold (1999) the column density and the ionization fraction are $\sim 10^{25} \mathrm{~cm}^{-2}$ and $10^{-12}$ at $R=1 \mathrm{AU}$. The growth time then is $\sim 2 \times 10^{7} B^{-2} \mathrm{~s}$ and, if the field is sufficiently strong ( $B \sim 1 \mathrm{G}$, the magnetic field found in meteorites), the baroclinic convection develope on timescales of the order of 1 year. The baroclinic convection in a magnetized gas might be qualitatively similar to the instability considered by Klahr (2004). Note, however, that a non-magnetic baroclinic instability requires a negative radial gradient of the entropy whereas the baroclinic instability in the presence of a magnetic field can occur even for positive gradients of entropy.

In magnetized regions with $a_{\mathrm{e}}>1$ other instabilities can manifest themselves if the wavelength of perturbations is sufficiently long. Consider the domains of different instabilities in the case when the wave vector is not perpendicular to $\boldsymbol{\Omega}$ and $\boldsymbol{B}$ so that $k_{z} \sim k$ and $(\boldsymbol{k} \cdot \boldsymbol{B}) \sim k B$. Then, the regions of instability are determined by two characteristic wavelengths, $\lambda_{\mathrm{H}}$ and $\lambda_{\mathrm{A}}$, corresponding to the conditions $\omega_{\mathrm{H}}=\Omega$ and $\omega_{\mathrm{A}}=\Omega$, respectively. They are

$\lambda_{\mathrm{H}}=3.1 \times 10^{12} B^{1 / 2} n_{\mathrm{e} 2}^{-1 / 2} P_{\mathrm{yr}}^{1 / 2} \mathrm{~cm}$,

$\lambda_{\mathrm{A}}=6.6 \times 10^{11} \mathrm{Bn}_{14}^{-1 / 2} P_{\mathrm{yr}} \mathrm{cm}$,

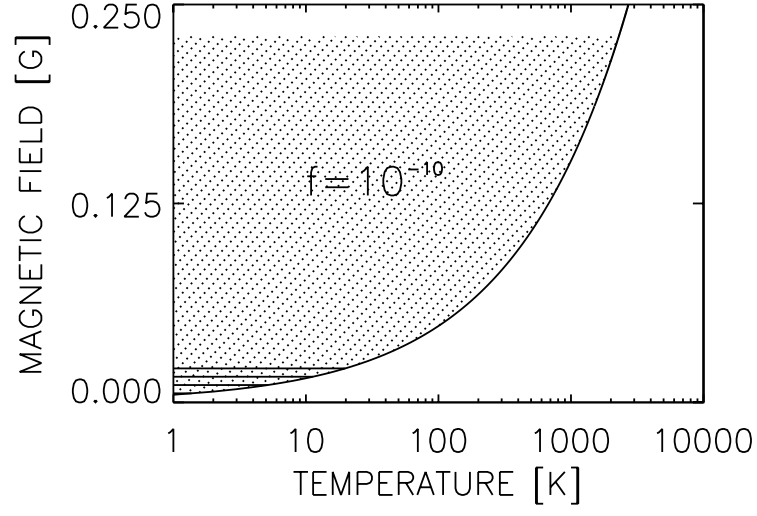

Fig. 2. The same as in Fig. 1 but only for $n_{14}=1$. The dotted area gives the domain of the Hall-driven shear instability for $f=10^{-10}$ while the hatched area gives the domain of the Hall-driven shear instability for $f=10^{-9}$.

and their ratio is given by

$\frac{\lambda_{\mathrm{A}}}{\lambda_{\mathrm{H}}} \sim\left(\frac{\Lambda}{a_{\mathrm{e}}}\right)^{1 / 2} \sim 0.21 B^{1 / 2} f_{-12}^{1 / 2} P_{\mathrm{yr}}^{1 / 2}$

with the Elsässer number $\Lambda=B^{2} /\left(\mu_{0} \rho \eta \Omega\right)$. If $\Lambda>a_{\mathrm{e}}$ (equivalent to $\Omega>\omega_{\mathrm{A}}>\sqrt{\omega_{\mathrm{H}} \Omega}$, slow rotation) the magnetic shear instability occurs with the growth rate (48). In the same domain, the baroclinic convection represented by criterion (16) may also arise.

If $\Lambda<a_{\mathrm{e}}$ (equivalent to $\Omega>\sqrt{\omega_{\mathrm{H}} \Omega}>\omega_{\mathrm{A}}$, fast rotation) then the shear-driven Hall instability can occur with the criteria given by Eqs. (20) and (38) (see Fig. 2). The growth rate of this instability depends on the Hall frequency and the shear (see Eq. (46)). If $\lambda<\lambda_{\mathrm{H}}$ then only criterion (15) of the baroclinic convection can be fulfilled. Note that this instability can also coexist with the shear-driven Hall instability if $\lambda>\lambda_{\mathrm{H}}$.

After Eqs. (57) and (58) with a theoretical disk model the radial wavelengths of different unstable regions can be determined as a function of the magnetic field and the radius $R$. After Eq. (59) for a given magnetic field the regions of shear-Hall instability and MRI can be found. The conditions, however, can be quite different in the midplane and the surface layers of protoplanetary disks. To illustrate the method we examine the midplane by means of the hydrostatic, radiative equilibrium model for T Tauri systems by Chiang \& Goldreich (1997). The surface mass density and the temperature there are given by

$\Sigma=10^{3} R_{13}^{-3 / 2}, \quad T \approx 1.3 \times 10^{2} R_{13}^{-3 / 4}$,

in cgs. Assuming the gas is isothermal one obtains for the gas density $\rho \simeq \Sigma / H$ with the scale height $H$. The model yields $H / R \approx 0.18 R_{13}^{2 / 7}$ so that the particle density in the midplane is $n_{14} \sim 3.5 R_{13}^{-39 / 14}$. On the other hand, the ionization fraction is approximately given by

$f=\frac{n_{\mathrm{e}}}{n} \approx \sqrt{\frac{\zeta}{\tilde{\beta} n}}$,

where $\tilde{\beta}=2 \times 10^{-6} T^{-1 / 2} \mathrm{~cm}^{3} / \mathrm{s}$ is the dissociative recombination rate (Igea \& Glassgold 1999) and $\zeta$ is the ionization rate. 
Assuming the ionization as mainly due to cosmic rays in the midplane (Salmeron \& Wardle 2004, 2005) and $\zeta \sim 10^{-17} \mathrm{~s}^{-1}$ one obtains

$f \sim 4 \times 10^{-13} \zeta_{-17}^{1 / 3} R_{13}^{1.18}$

Note that in the inner region with a large column density $\left(>10^{2} \mathrm{~g} / \mathrm{cm}^{2}\right)$, the ionization rate at the midplane can be reduced (Umebayashi \& Nakano 1981). Likely, at $\Sigma>$ $150 \mathrm{~g} / \mathrm{cm}^{2}$, electrons and photons become the main ionization sources, and the attenuation length is $\sim 100 \mathrm{~g} / \mathrm{cm}^{2}$ for the ionization rate and $\sim 200 \mathrm{~g} / \mathrm{cm}^{2}$ for the ionization fraction. At $\Sigma>1100 \mathrm{~g} / \mathrm{cm}^{2}$, the ionization by radioactive elements is more efficient than by the energetic particles. Therefore, in the region with a very high column density, $\Sigma \geq 10^{2}-10^{3} \mathrm{~g} / \mathrm{cm}^{2}$ or $R_{13}<4.7$ in the model given by Eq. (60), the ionization fraction given by Eq. (61) is likely overestimated. The region with $R_{13}<4.7$ corresponds approximately to the dead zone.

With Eqs. (57) and (58) the characteristic wavelengths $\lambda_{\mathrm{A}}$ and $\lambda_{\mathrm{H}}$ can be computed as

$\lambda_{\mathrm{A}} \approx 3.5 \times 10^{11} B R_{13}^{2.89} \mathrm{~cm}$

and

$\lambda_{\mathrm{H}} \approx 2.6 \times 10^{12} B^{1 / 2} \zeta_{-17}^{-1 / 4} R_{13}^{1.55} \mathrm{~cm}$.

They are equal for

$R_{13}=4.5 B^{-0.37} \zeta_{-17}^{0.18}$,

with $B$ measured in Gauss. Hence, the standard regime of the MRI with the growth rate (48) occurs only in the outer region for $R_{13}>4.5 B^{-0.37} \zeta_{-17}^{-0.18}$. In the inner region with $R_{13}<4.5 B^{-0.37} \zeta_{-17}^{-0.18}$, the shear-Hall instability exists with the growth rate (46). As noted above neither of these instabilities can occur in the dead zone where the Ohmic dissipation dominates. The boundary between the regions with different regimes of instability is larger than the radius of the dead zone if $B<\zeta_{-17}^{-1 / 2}$. Since outside the dead zone $\zeta_{-17} \sim 1$, the region where the Hall-driven instability could be dominant exists only if $B<1 \mathrm{G}$. For fields parallel to the rotation axis Rüdiger \& Kitchatinov (2005) find the minimum magnetic amplitude of $10^{-3} \mathrm{G}$ for the shear-Hall instability. It seems from Eq. (65), however, that this region is not very extended for reasonable values of the magnetic field in protoplanetary disks. Nevertheless, the shear-Hall instability can occur in the layers above the midplane where the ionization is higher.

In the present paper the behavior of only axisymmetric perturbations is addressed. It is clear, however, that the results can be applied to nonaxisymmetric perturbations with an azimuthal wavelength much longer than the vertical or radial ones, $k_{\phi}<\min \left(k_{R}, k_{z}\right)$. The turbulence that could be generated by the considered instabilities may be strongly anisotropic in the $(R, z)$-plane because the instability criteria are sensitive to the direction of the wave vector. However, the generated turbulence may be efficient in the radial transport of angular momentum.

Acknowledgements. The financial support by the Deutsche Forschungsgemeinschaft (436 RUS 113/559) is cordially acknowledged. V.U. also acknowledges financial support by the Russian Foundation of Basic Research (grant 04-02-16243).

\section{References}

Aleksandrov, A., et al. 1985, Mathematics: Its Content, Methods, and Meaning (Cambridge: MIT)

Arlt, R., \& Rüdiger, G. 2001, A\&A, 374, 1035

Balbus, S. A. 1995, ApJ, 453, 380

Balbus, S. 2000, ApJ, 534, 420

Balbus, S. A., \& Hawley, J. F. 1991, ApJ, 376, 214

Balbus, S., \& Terquem, C. 2001, ApJ, 552, 235

Brandenburg, A., Nordlund, Å., Stein, R., et al. 1995, ApJ, 446, 741

Chiang, E., \& Goldreich, P. 1997, ApJ, 480, 368

Curry, C., Pudritz, R., \& Sutherland, P. 1994, ApJ, 434, 206

Curry, C., \& Pudritz, R. 1995, ApJ, 453, 697

Draine, B., Roberge, W., \& Dalgarno, A. 1983, ApJ, 264, 485

Drecker, A., Rüdiger, G., \& Hollerbach, R. 2000, MNRAS, 317, 45

Dubrulle, B., Marié, L., Normand, Ch., et al. 2005, A\&A, 429, 1

Gammie, C. 1996, ApJ, 457, 355

Hawley, J. F., Gammie, C. F., \& Balbus, S. A. 1995, ApJ, 440, 742

Igea, J., \& Glassgold, A. 1999, ApJ, 518, 848

Jin, L. 1996, ApJ, 457, 798

Kaisig, M., Tajima, T., \& Lovelace, R. 1992, ApJ, 386, 83

Keppens, R., Casse, F., \& Goedbloed, J. 2002, ApJ, 569, L121

Kitchatinov, L. L., \& Rüdiger, G. 1997, MNRAS, 286, 757

Klahr, H. 2004, ApJ, 606, 1070

Knobloch, E., \& Spruit, H. 1986, A\&A, 166, 359

Kumar, S., Coleman, C., \& Kley, W. 1994, MNRAS, 266, 379

Kurzweg, U. 1963, JFM, 17, 52

Matsumoto, R., \& Tajima, T. 1995, ApJ, 445, 767

Rüdiger, G., \& Kitchatinov, L. L. 2005, A\&A, 434, 629

Rüdiger, G., \& Shalybkov, D. A. 2004, Phys. Rev. E, 69, 016303

Salmeron, R., \& Wardle, M. 2004, Ap\&SS, 292, 451

Salmeron, R., \& Wardle, M. 2005, MNRAS

Sano, T., Inutsuka, S. I., \& Miyama, S. M. 1998, ApJ, 506, L57

Sano, T., \& Stone, J. 2002a, ApJ, 570, 314

Sano, T., \& Stone, J. 2002b, ApJ, 577, 534

Shalybkov, D. A., \& Urpin, V. 1995, MNRAS, 273, 643

Spitzer, L. 1978, Physical Processes in the Interstellar Medium (New York: Wiley)

Torkelsson, U., Brandenburg, A., Nordlund, Å., \& Stein, R. 1996, Astrophys. Lett. \& Comm., 34, 383

Umebayashi, T., \& Nakano, T. 1981, PASJ, 33, 617

Urpin, V. 1996, MNRAS, 280, 149

Urpin, V. 2003, A\&A, 404, 397

Urpin, V., \& Brandenburg, A. 1998, MNRAS, 294, 399

Velikhov, E. P. 1959, Sov. Phys. JETP, 9, 995

Wardle, M. 1999, MNRAS, 307, 849

Wardle, M., \& Ng, C. 1999, MNRAS, 303, 239

Zhang, W., Diamond, P., \& Vishniac, E. 1994, ApJ, 420, 705 